\title{
Refractive Index of Natural Rubber for Different Wavelengths ${ }^{1}$
}

\author{
By Lawrence A. Wood and Leroy W. Tilton
}

\begin{abstract}
The refractive indices of a prism of natural rubber have been measured at five different wavelengths in the visible region by the use of a spectrometer. Values af the rate of change of index with temperature at each wavelength have been computed for the range $19.5^{\circ}$ to $30.3^{\circ} \mathrm{C}$. The Eykman equation in differential form is used to calculate the expansivity from the index and its rate of change with temperature. It is concluded that the two-constant Cauchy and Sellmeier dispersion equations are inadequate to represent the data. The dispersion observed for rubber is found to be essentially the same as that of hydrocarbons of similar structure but of low molecular weight.
\end{abstract}

\section{Introduction}

Published values of the refractive index of natural rubber, with very few exceptions, have been concerned with the value at the wavelength of the sodium $D$-lines (5,893 angstroms). This paper presents the results of measurements by the spectrometer method at this wavelength and four other wavelengths in the visible spectrum. Observations of the effect of temperature on index are also made. The constants of several different types of dispersion equations are evaluated in order to obtain an equation to represent the data. The dispersion observed for rubber is compared with that of hydrocarbons of low molecular weight.

The experimental portion of this work was conducted in 1939, and some of the results presented in graphical form in a general paper by one of the present authors [1]. ${ }^{2}$ The war prevented further work on this problem until recently.

\section{Method of Measurement}

The well-known and conventional method of measurement of refractive index by observations of the angle of minimum deviation produced by a

\footnotetext{
1 This paper was presented on June 24, 1948, at the Rubber Technology Conference, London, England, sponsored by the Institution of the Rubber Industry. It has been published as paper No. 31, page 142, in the Proceedings of the Rubber Technology Conference; London 1948.

2 Figures in brackets indicate the literature references at the end of this paper.
}

prism of known angle does not seem to have been previously applied to rubber. This very direct method commonly yields results of higher precision and accuracy than can be obtained by available commercial refractometers since it is free from many of the complications and limitations inherent in comparison methods by critical-angle refractometry.

The rubber prisms required were made by molding the rubber between two plane glass plates inclined at an angle. A mold of 1-in., cold-rolled steel bars fastened together by bolts had an opening in which from one to six steel wedges could be placed, thereby fixing the angle of the prism at about $10^{\circ}$ or some multiple of $10^{\circ}$. The rubber was molded against two glass plates 20 by 10 by $3.5 \mathrm{~mm}$, which were left in position after the molding. The glass plates were special "plane parallels," that is, they had faces which were plane and parallel to each other to within a few wavelengths of light, as evidenced by comparison with optical flats and by examination of the interference phenomenon known as Haidinger's rings. Since their faces were parallel, the collimated light beam in its passage through the glass underwent no deviation or dispersion, and the observed refraction was due entirely to the wedge of rubber between the plates.

The pale crepe rubber was milled only very slightly, a few passages through the warm rolls 
being found sufficient to make possible the production of a sheet about $1 \mathrm{~cm}$ in thickness and relatively free from entrapped air. A specimen was cut very roughly to shape and inserted in the mold just described. The molding was performed in a steam vulcanizing press at a temperature of $150^{\circ} \mathrm{C}$ for about $30 \mathrm{~min}$.

When the prism was placed on the spectrometer table it was observed that the refracting angle showed a systematic change with time, a decrease of several minutes of angle a day being usually observed. This circumstance, arising no doubt from the plastic flow of the unvulcanized rubber, made it necessary to measure the prism angle before and after each set of measurements of the angle of deviation. The measurements of the refracting angle were made by means of reflections from the glass surfaces by the use of the spectrometer telescope, which was essentially autocollimating. As pointed out in a previous publication [2] the commonly used "split beam" method of measurement of refracting angle is never advisable. In the present instance additional difficulties would have arisen because of the finite thickness of the glass plates in contact with the rubber.

The spectrometer, which was manufactured by the Société Genevoise, has been described in detail in an earlier publication [3]. It has a circle $308 \mathrm{~mm}$ in diameter and is graduated to 5-min intervals. The use of micrometer microscopes allowed angles to be read to the nearest second.

The prism was set at the angle of minimum deviation in each case and the refractive index $n$ computed from the usual spectrometer equation

$$
n=\frac{\sin 1 / 2(A+D)}{\sin 1 / 2 A},
$$

where $A$ is the refracting angle of the prism and $D$ the angle of minimum deviation.

The light sources used were a sodium arc, a hydrogen discharge tube, and a mercury vapor lamp. The wavelengths of the spectral lines utilized are as follows:

\begin{tabular}{|c|c|c|}
\hline $\begin{array}{l}\text { Wave- } \\
\text { length }\end{array}$ & Source & $\begin{array}{l}\text { Desig- } \\
\text { nation }\end{array}$ \\
\hline Angstroms & Hydrogen (alnha lin & $C$ \\
\hline 5892.62 & $\begin{array}{l}\text { Sodium (weighted mean of two } \\
\text { lines). }\end{array}$ & $D$ \\
\hline $\begin{array}{l}5460.74 \\
4861.33\end{array}$ & $\begin{array}{l}\text { Mercury } \\
\text { Hydrogen (beta line) }\end{array}$ & $\stackrel{e}{F}$ \\
\hline 4358.34 & Mercury & $g$ \\
\hline
\end{tabular}

The measurements with the $g$-line were made with more difficulty than those at longer wavelengths because of the greatly reduced transmission of light and the increased amount of light scattered by the sample in this region. Measurements of the transmission and scattering have been given in the earlier paper on the optical properties of rubber [1].

\section{Results}

Table 1 gives the results of the measurements made with prism 1 , having a refracting angle near $20^{\circ}$. More measurements were made with this prism than with any of several others made since it appeared to possess the best optical properties. Measurements were made at all five wavelengths, and a stirred air-bath in a water-jacketed constanttemperature prism housing equipped with a thermostat was employed to obtain values at $19.5^{\circ}$ and $39.3^{\circ} \mathrm{C}$. The temperatures could be measured to the nearest hundredth of a degree. At least $1 \mathrm{hr}$ at any given temperature was allowed to elapse before readings of index were made.

The data shown in table 1 for each wavelength were treated by the methods of linear regression, or "least squares," as outlined in books on statistical methods, for example, the text of Snedecor [4]. Inherent in this treatment is the usual assumption that there are no errors in the determination of the independent variable, the temperature in this case. By these methods values were obtained for the indices at $25^{\circ} \mathrm{C}$, their standard deviations, and the rates of change of indices with temperature and their standard deviations. 
TABLE 1. Data obtained with prism 1

\begin{tabular}{|c|c|c|c|c|c|c|c|c|c|c|c|}
\hline \multirow{2}{*}{\multicolumn{2}{|c|}{1939}} & \multicolumn{3}{|c|}{ Angle } & \multirow{2}{*}{$\begin{array}{l}\text { Temper- } \\
\text { ature }\end{array}$} & \multirow{2}{*}{$n_{c}$} & \multirow{2}{*}{$n_{D}$} & \multirow{2}{*}{$n$. } & \multirow{2}{*}{$n_{F}$} & \multirow{2}{*}{$n_{\theta}$} & \multirow{2}{*}{$n_{F}-n_{C}$} \\
\hline & & Degrees & Minutes & Seconds & & & & & & & \\
\hline \multirow{6}{*}{ July } & 17 & & 55 & 25 & $\begin{array}{c}{ }^{\circ} \mathrm{C} \\
28,7\end{array}$ & 1.51365 & 1. 51737 & & 1. 52679 & & 0.01314 \\
\hline & 18 & 20 & 52 & 15 & 28.0 & 1. 51415 & 1,51793 & & 1. 52732 & & 01317 \\
\hline & 18 & 20 & 52 & 2 & 28.5 & 1. 51409 & 1. 51782 & & (n) & & \\
\hline & 19 & 20 & 50 & 24 & 28.4 & 1.51390 & 1. 51765 & & 1. 52704 & & .01314 \\
\hline & 19 & 20 & 50 & 26 & 28.5 & 1. 51408 & 1. 51773 & 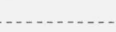 & 1. 52725 & & .01317 \\
\hline & 19 & 20 & 50 & 17 & 28.7 & 1. 51411 & 1,51781 & & 1. 52720 & & .01309 \\
\hline \multirow[t]{8}{*}{ Sept. } & 2 & 20 & 39 & 48 & 28.4 & 1. 51407 & 1. 51793 & 1. 52121 & 1. 52727 & 1. 53510 & .01320 \\
\hline & 5 & 20 & 39 & 30 & 26.5 & 1. 51488 & 1. 51868 & 1. 52196 & 1. 52809 & 1. 53574 & .01321 \\
\hline & 5 & 20 & 39 & 37 & 27.1 & 1.51475 & 1. 51850 & 1. 52179 & 1. 52790 & 1. 53557 & .01315 \\
\hline & 6 & 20 & 39 & 31 & 26.9 & 1. 51474 & 1. 51847 & 1. 52172 & 1. 52791 & 1. 53554 & .01317 \\
\hline & 7 & 20 & 45 & 52 & 39.3 & 1. 51013 & 1. 51385 & 1. 51707 & 1. 52324 & 1. 53088 & .01311 \\
\hline & 7 & 20 & 45 & 52 & 39.3 & 1. 51012 & 1. 51382 & 1. 51704 & 1. 52318 & 1. 53082 & .01306 \\
\hline & 11 & 20 & 34 & 45 & 19.5 & 1. 51737 & 1. 52113 & 1. 52440 & 1. 53062 & 1. 53835 & .01325 \\
\hline & 11 & 20 & 34 & 45 & 19.5 & 1. 51739 & 1. 52111 & 1. 52432 & 1. 53066 & 1. 53832 & .01327 \\
\hline \multicolumn{5}{|c|}{ Computed from above data at } & 25.0 & 1. 515345 & 1. 519093 & 1. 522415 & 1. 528536 & 1. 536292 & .013193 \\
\hline
\end{tabular}

Some observations were also made on prism 2, a prism of less satisfactory optical quality than prism 1. The results are shown in table 2. The second prism had a refracting angle near $10^{\circ}$ and of course did not produce as large an angle of deviation as prism 1. Observations were made with this prism for the sodium $D$-lines, the hydrogen $C$-line, and the hydrogen $F$-line only. The temperature range covered was likewise much smaller than previously.

Table 3 presents a comparison of the results obtained on the two prisms. As would be ex- pected, the standard deviations obtained with prism 2 are considerably larger than those found with prism 1. In the last column $\left(n_{F}-n_{C}\right)$ is shown as calculated from the individual observations shown in tables 1 and 2 . The desirability of computing the difference in this manner is demonstrated by the fact that the standard deviations for it are considerably smaller than those for the indices at the individual wavelengths.

The differences between the values of index at each wavelength for the two prisms can be seen to be of no significance when they are compared with

TABLE 2. Data obtained with prism 2

\begin{tabular}{|c|c|c|c|c|c|c|c|c|c|}
\hline \multirow{2}{*}{\multicolumn{2}{|c|}{1939}} & \multicolumn{3}{|c|}{ Angle } & \multirow{2}{*}{$\begin{array}{c}\text { Tem- } \\
\text { peratule }\end{array}$} & \multirow{2}{*}{$n_{C}$} & \multirow{2}{*}{$n_{D}$} & \multirow{2}{*}{$n_{\boldsymbol{F}}$} & \multirow{2}{*}{$n_{P}-n_{C}$} \\
\hline & & Degrees & Minutes & Seconds & & & & & \\
\hline \multirow{3}{*}{$\begin{array}{rr}\text { July } & 2 \\
2 & 2 \\
2\end{array}$} & 22 & 9 & 58 & 16 & 28.2 & 1. 51420 & 1. 51792 & 1. 52735 & 0.01315 \\
\hline & 24 & 9 & 58 & 3 & 29.0 & 1. 51408 & 1. 51779 & 1. 52722 & .01314 \\
\hline & 25 & 9 & 57 & 59 & 29.0 & 1. 51394 & 1. 51766 & 1. 52712 & .01318 \\
\hline \multirow[t]{6}{*}{ Aug. } & 17 & 10 & 4 & 35 & 32.3 & 1. 51278 & 1. 51647 & 1. 52581 & .01303 \\
\hline & 18 & 10 & 4 & 48 & 32.3 & 1. 51286 & 1. 51660 & 1. 52601 & .01315 \\
\hline & 18 & 10 & 4 & 47 & 32.3 & 1. 51295 & 1. 51659 & 1. 52605 & .01310 \\
\hline & 28 & 10 & 2 & 56 & 28.2 & 1. 51438 & 1. 51806 & 1. 52743 & .01305 \\
\hline & 30 & 10 & 2 & 36 & 27.2 & 1. 51466 & 1. 51836 & 1. 52772 & .01306 \\
\hline & 31 & 10 & 2 & 19 & 26.5 & 1. 52482 & 1. 51860 & 1. 52794 & .01312 \\
\hline \multicolumn{5}{|c|}{ Computed from above data at... } & 25.0 & 1. 515365 & 1. 519095 & 1. 528470 & .013117 \\
\hline
\end{tabular}


TABLE 3. Comparison of results obtained with the two different prisms

\begin{tabular}{|c|c|c|c|c|c|c|}
\hline & $C$ & $D$ & $e$ & $F$ & $g$ & $(F-C)^{\mathrm{a}}$ \\
\hline Index $n^{25}$ for prism $1 \ldots$ & 1. 515345 & 1. 519093 & 1. 522415 & 1. 528536 & 1. 536292 & 0.013193 \\
\hline Standard deviation of $n^{25}$ & $37.4 \times 10^{-6}$ & $35.2 \times 10^{-6}$ & $63.6 \times 10^{-6}$ & 43. $2 \times 10^{-6}$ & $49.3 \times 10^{-6}$ & $10.5 \times 10^{-6}$ \\
\hline Index $n^{25}$ for prism $2 \ldots \ldots$ & 1. 515365 & 1. 519095 & ........... & 1. 528470 & ........... & 0.013117 \\
\hline Standard deviation of $n^{25}$ & $94.8 \times 10^{-6}$ & $152 \times 10^{-6}$ & & $147 \times 10^{-6}$ & & $42.5 \times 10^{-6}$ \\
\hline Difference between indices of prism 2 and prism 1 ... & $20 \times 10^{-6}$ & $2 \times 10^{-6}$ & - & $-66 \times 10^{-6}$ & $\ldots$ & $-76 \times 10^{-6}$ \\
\hline$d n / d T$ for prism 1 & $-367.7 \times 10^{-6}$ & $-369.9 \times 10^{-6}$ & $-370.1 \times 10^{-6}$ & $-376.5 \times 10^{-6}$ & $-378.2 \times 10^{-6}$ & $-8.8 \times 10^{-6}$ \\
\hline Standard deviation of $d n / d T$ for prism $1 \ldots \ldots$ & 5. $90 \times 10^{-6}$ & 5. $54 \times 10^{-6}$ & $8.11 \times 10^{-6}$ & $6.62 \times 10^{-6}$ & 6. $29 \times 10^{-6}$ & $1.6 \times 10^{-6}$ \\
\hline$d n / d T$ for prism 2 & $-341 \times 10^{-6}$ & $-345 \times 10^{-6}$ & & $-340 \times 10^{-6}$ & & $-1.8 \times 10^{-6}$ \\
\hline Standard deviation of $d n / d T$ for prism 2 & 19. $2 \times 10^{-6}$ & $30.7 \times 10^{-6}$ & & $29.7 \times 10^{-6}$ & & $8.6 \times 10^{-6}$ \\
\hline $\begin{array}{l}\text { Difference between values of } d n / d T \text { of prism } 2 \text { and } \\
\text { prism } 1\end{array}$ & $+26.7 \times 10^{-6}$ & $+24.9 \times 10^{-6}$ & & $+36.5 \times 10^{-6}$ & & $+7.0 \times 10^{-6}$ \\
\hline
\end{tabular}

a The values in this column have been calculated directly from the observations shown in tables 1 and 2 , and are not obtained from differences of preceding columns of table 3 .

the standard deviations. The differences are less than the standard deviation of prism 2 for each of the three spectral lines, and even for $\left(n_{F}-n_{C}\right)$ the difference is less than twice the standard deviation. Similarly, the differences in $d n / d T$ are not significant, since they are less than twice the standard deviation.

Since the precision of the values obtained with prism 1 was much greater than that obtained with prism 2 and more observations were made with it, the remainder of the paper will be concerned only with the values obtained on prism 1 .

It should be clearly recognized that the present work did not include a study of the dependence of refractive index on composition. The rubber used to make the second prism probably came from the same bale as that used to make the first. For simplicity the results have been treated as if the sample were pure rubber hydrocarbon, whereas it is only 93 to 94 percent hydrocarbon and is known to contain resins in solution and proteins, salts, and other foreign material dispersed in it. Hence the number of significant figures used here shows only the precision of the present measurements of a sample of given composition. Earlier work $[5,6]$ has shown little variation with composition in the fourth-place index values, but further studies would be required to show the effect of impurities on fifth-place and sixth-place values.

\section{Discussion}

The results of measurements of refractive index for the $D$-line have been summarized in a paper presented at the Rubber Technology Conference in 1938 [7]. The most reliable value of $n_{D}^{25}$ was judged to be 1.5190, found by McPherson and Cummings [6]. This is in very good agreement with that found in the present work, namely, 1.519093 .

McPherson and Cummings also made measurements with a Pulfrich refractometer and reported an exceedingly large value for the dispersion, $n_{F}-n_{C}$. In the course of the present work the original data of McPherson and Cummings were reexamined and it was discovered that on account of a clerical error incorrect values were given for the refractive index of the $F$-line and consequently for the dispersion. The corrected results of McPherson and Cummings are as follows:

\begin{tabular}{|c|c|c|}
\hline Temperature & $24.5^{\circ} \mathrm{C}$. & $25.0^{\circ} \mathrm{C}$. \\
\cline { 1 - 2 } & & \\
$n_{C} \ldots \ldots$ & 1.5153 & 1.5151 \\
$n_{D} \ldots \ldots$ & 1.5188 \\
$n_{F}-n_{C} \ldots \ldots$ & 1.5283 & 1.5281 \\
& 0.0130 & 0.0130 \\
\hline
\end{tabular}

These values are lower than those given in the present work by 2 to 4 units in the fourth decimal place.

A summary of previous values of the rate of change of index ( $D$-line) with temperature is as follows:

\begin{tabular}{|c|c|c|}
\hline Author & Year & $d n / d T$ \\
\hline McPherson [5] & 1932 & $360 \times 10^{-6}$ \\
\hline Kirchof $[8]$ & 1932 & 369 \\
\hline McPherson and Cummings [6]_ & 1935 & 350 \\
\hline Present work & ...- & 369.9 \\
\hline
\end{tabular}


In a previous publication [7] it has been shown that the empirical Eykman equation, $\left(n^{2}-1\right) /$ $(n+0.4)=R d$, where $d$ is the density and $R$ a constant, can be differentiated to yield the following equation

$$
\frac{d n}{d T}=-\frac{1}{\frac{2 n}{n^{2}-1}-\frac{1}{n+0.4}}\left(\frac{1}{V} \frac{d V}{d T}\right) .
$$

Here $V$ is the volume of a given mass of the material, so that $(1 / V)(d V / d T)$ is its volume expansivity. It has now been found that the coefficient by which the expansivity is multiplied in the righthand member of this equation can be represented to within about 0.2 percent over the complete range of normal refractive indices, namely from 1.33 to 1.7 , by the expression $(1.10440 n-1.12226)$. Thus the equation becomes

$$
\frac{d n}{d T}=-(1.10440 n-1.12226)\left(\frac{1}{V} \frac{d V}{d T}\right) .
$$

Of the three observable quantities involved in this equation it is considered that the experimental uncertainty is greatest in the value of the expansivity. Consequently, the expansivity has been calculated from the optical data by taking the ratio of $(d n / d T)$ to $(1.10440 n-1.12226)$ for each of the spectral lines. The following results were obtained

\begin{tabular}{|c|c|}
\hline Spectral line & $\begin{array}{c}\text { Calculated } \\
\text { expansivity }\end{array}$ \\
\cline { 2 - 2 } $\mathrm{C}$ & $667 \times 10-6$ \\
$D$ & 666 \\
$e$ & 662 \\
$\mathrm{~F}$ & 665 \\
$g$ & 658 \\
\hline
\end{tabular}

The mean value, $664 \times 10^{-6}$ (deg. C $)^{-1}$, is in excellent agreement with values directly observed $[7,9]$.

By inserting this mean value for the expansivity and the value for $n^{25}$ in eq 3 one then has $d n / d T=-\left(1.10440 n^{25}-1.12226\right)\left(664 \times 10^{-6}\right)$.

The values of the right-hand member of this equation are compared with $d n / d T$ as directly observed

\begin{tabular}{|c|c|c|c|}
\hline \multirow{2}{*}{$\begin{array}{l}\text { Spectral } \\
\text { line }\end{array}$} & \multicolumn{2}{|c|}{$d n / d T$} & \multirow{2}{*}{ Difference } \\
\hline & Calculated & Observed & \\
\hline $\begin{array}{l}C \\
D \\
e \\
F \\
g\end{array}$ & $\begin{array}{l}\text { 366. } 1 \times 10^{-6} \\
368.8 \\
371.2 \\
\text { 375. } 7 \\
381.4\end{array}$ & $\begin{array}{l}\text { 367. } 7 \times 10^{-6} \\
369.9 \\
370.1 \\
376.5 \\
378.2\end{array}$ & $\begin{array}{l}-1.6 \times 10^{-6} \\
-1.1 \\
+1.1 \\
-0.8 \\
+3.2\end{array}$ \\
\hline
\end{tabular}
in the following tabulation
The agreement is very satisfactory.

The molecular resractivity can be calculated by the Lorenz-Lorentz relation from the present data by taking the value $0.906 \mathrm{~g} / \mathrm{cm}^{3}$ for the density at $25^{\circ} \mathrm{C}$. of purified natural rubber $[5,7]$. As pointed out in the earlier paper [1] the result, 22.82 , is in sufficiently good agreement with values obtained by taking the sum of atomic refractivities to afford optical confirmation of the existence of a $\mathrm{C}_{5} \mathrm{H}_{8}$ group containing one double bond as the unit group in rubber. It would be desirable to apply the methods outlined by Taylor, Pignocco, and Rossini [10] to a more detailed study of the data given in the present paper.

\section{Comparison of Refractivity Intercept and Specific Dispersion With Values for Other Hydrocarbons}

The refractivity intercept, $n_{D}-d / 2$, and specific dispersion, $10^{4}\left(n_{F}-n_{C}\right) / d$, where $d$ is the density, have been used for some years to distinguish between different classes of hydrocarbons [11]. Values for these quantities applicable to hydrocarbons with not more than nine carbon atoms are given in Circular C461 of the National Bureau of Standards, entitled Selected values of properties of hydrocarbons, by Rossini and coworkers [12]. The lowest and the highest values found for each class of hydrocarbon, together with the mean of the two, are listed in table 4 for comparison with the refractivity intercept and the specific dispersion for rubber. In these computations the value $0.906 \mathrm{~g} / \mathrm{cm}^{3}$ has again been used as the density at $25^{\circ} \mathrm{C}$ of purified natural rubber.

From the comparison shown in table 4 one can see that both the refractivity intercept and specific dispersion of rubber are somewhat above the corresponding values for the monoolefins but not nearly as high as those for the diolefins with 
conjugated double bonds. This is exactly what would be expected from the structure of the rubber polymer. The double bonds in rubber are separated by four skeletal carbon atoms rather than by two carbon atoms as in a conjugated system. It has already been suggested [13] that this fact gives rise to a slight degree of double bond character in the single bond half-way between two double bonds.

TABLE 4. Comparison of values of refractivity intercept and specific dispersion with those found in NBS Circular 461, Selected Values of Properties of Hydrocarbons, by Rossini and coworkers [12]

\begin{tabular}{|c|c|c|c|c|c|c|}
\hline & \multirow{2}{*}{\multicolumn{3}{|c|}{$\frac{\text { Refractivity intercept }}{n_{D}^{25}-d / 2}$}} & \multirow{2}{*}{\multicolumn{3}{|c|}{$\begin{array}{c}\text { Specific dispersion } \\
10^{4}\left(n_{F}^{25}-n_{C}^{25}\right) / d\end{array}$}} \\
\hline & & & & & & \\
\hline & Low & High & Mean a & Low & High & Mean a \\
\hline \multicolumn{7}{|l|}{ Paraffins: } \\
\hline $\mathrm{C}_{5} \mathrm{H}_{12} \ldots$ & 1.0436 & 1. 0441 & 1.04385 & 98 & 99 & 98.5 \\
\hline $\mathrm{C}_{6} \mathrm{H}_{14 \ldots}$ & 1. 0437 & 1.0448 & 1.04425 & 97 & 100 & 98.5 \\
\hline $\mathrm{C}_{7} \mathrm{H}_{16 \ldots}$ & 1. 0439 & 1. 0454 & 1.04465 & 96 & 100 & 98 \\
\hline $\mathrm{C}_{8} \mathrm{H}_{18 \ldots}$ & 1. 0437 & 1. 0458 & 1.04475 & 96 & 100 & 98 \\
\hline $\mathrm{C}_{9} \mathrm{H}_{20 \ldots . . .}$ & 1. 0420 & 1. 0470 & 1.0445 & 94 & 101 & 97.5 \\
\hline \multicolumn{7}{|l|}{ Monoolefins: } \\
\hline $\mathrm{C}_{5} \mathrm{H}_{10} \ldots$ & 1.050 & 1. 056 & 1.053 & 128 & 135 & 131.5 \\
\hline $\mathrm{C}_{6} \mathrm{H}_{12} \ldots$ & 1.049 & 1. 058 & 1.0535 & 122 & 132 & 127 \\
\hline $\mathrm{C}_{7} \mathrm{H}_{14 \ldots} \ldots$ & 1.048 & 1.057 & 1.0525 & 118 & 130 & 124 \\
\hline $\mathrm{C}_{8} \mathrm{H}_{16} \ldots$ & 1.050 & 1.055 & 1.0525 & 116 & 127 & 121.5 \\
\hline \multicolumn{7}{|l|}{$\begin{array}{l}\text { Diolefins with con- } \\
\text { jugated double } \\
\text { bonds: }\end{array}$} \\
\hline $\mathrm{C}_{5} \mathrm{H}_{8} \ldots$ & 1.080 & 1.091 & 1.0855 & 225 & 225 & 225 \\
\hline $\mathrm{C}_{6} \mathrm{H}_{10} \ldots$ & 1.075 & 1. 091 & 1.083 & 225 & 225 & 225 \\
\hline \multicolumn{7}{|l|}{$\begin{array}{l}\text { Diolefins with ad- } \\
\text { jacent } \\
\text { bonds: }\end{array}$} \\
\hline $\mathrm{C}_{5} \mathrm{H}_{8}$ & 1.06 & 1.074 & 1.067 & - & -- & -... \\
\hline $\mathrm{C}_{6} \mathrm{H}_{10} \ldots \ldots$ & 1. 054 & 1. 069 & 1.0615 & -- & -- & $\cdots$ \\
\hline $\begin{array}{c}\text { Other diolefins: } \\
\mathrm{C}_{5} \mathrm{H}_{8}\end{array}$ & & & 1.057 & & & \\
\hline $\begin{array}{l}\mathrm{C}_{5} \mathrm{H}_{8} \mathrm{H}_{10} \\
{ }_{10}\end{array}$ & $\begin{array}{l}1.057 \\
1.057\end{array}$ & $\begin{array}{l}1.057 \\
1.062\end{array}$ & $\begin{array}{l}1.008 \\
1.0595\end{array}$ & -- & $\begin{array}{l}-- \\
--\end{array}$ & -..- \\
\hline $\begin{array}{l}\text { Rubber } b \text { : } \\
\left(\mathrm{C}_{5} \mathrm{H}_{8}\right)_{p}\end{array}$ & $\cdots$ & $-\cdots$ & 1.066 & -- & -- & 145.6 \\
\hline
\end{tabular}

${ }^{a}$ Mean of low and high values.

$b$ The number of $\left(\mathrm{C}_{5} \mathrm{H}_{8}\right)$-units, or degree of polymerization, is denoted by $p$.

\section{Dispersion Equations}

From the data obtained in the present work it is possible to calculate the constants in a dispersion equation, which gives the relation between refractive index and wavelength. From a consideration of standard deviations one can draw conclusions regarding the validity of a partıcular form of dispersion equation. The present work shows that neither the Cauchy dispersion equation nor the Sellmeier dispersion equation is adequate to represent the observed data if the number of parameters is limited to two.

The two-constant Cauchy dispersion equation [11] is usually written

$$
n=A_{1}+B_{1} / \lambda^{2}=A_{1}+B_{1} \nu^{2} .
$$

Here $n$ is the refractive index, $\lambda$ is the wavelength, and $\nu$ its reciprocal, the wavenumber of the light. $A_{1}$ and $B_{1}$ are constants characteristic of the medium.

The constants in the Cauchy equation were evaluated at the five different wavelengths from the index for prism 1 by the usual methods of linear regression. The following equation was thus obtained

$$
n=1.498648+71.16 \times 10^{-6} / \lambda^{2} .
$$

A comparison of observed values with those calculated from this equation is given in table 5 . It will be seen that the differences are considerably larger than the corresponding standard deviations of the observations. The observed variance in the Cauchy equation values yields a standard deviation of $176 \times 10^{-6}$, which can be compared with the average standard deviation of an observed value, namely, $46 \times 10^{-6}$. Consequently, it is concluded that the Cauchy dispersion equation with two parameters does not adequately represent the observations.

The single-term Sellmeier dispersion equation [11] is usually written

$$
n^{2}-1=C /\left(\nu_{0}^{2}-\nu^{2}\right),
$$

where $C$ and $\nu_{0}$ are constants. For purposes of calculation this equation may be written in the following form

$$
1 /\left(n^{2}-1\right)=A_{2}+B_{2} \nu^{2} .
$$

The constants have been evaluated from the index values for prism 1, to yield the following equation

$$
1 /\left(n^{2}-1\right)=0 \cdot 800139-123.09 \times 10^{-12} \nu^{2} .
$$

Table 5 also shows the comparison of observed values with those calculated from this equation. 
TABLE 5. Comparison of dispersion equations

\begin{tabular}{|c|c|c|c|c|c|c|}
\hline 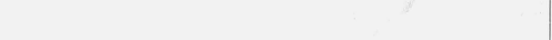 & $C$ & $D$ & $e$ & $F$ & $g$ & $F-C$ \\
\hline $\begin{array}{l}\text { Observed value } n^{25} \\
\text { Standard deviation } \\
\text { Calculated from Cauchy equation } \\
\text { Difference between observed and calculated values.- } \\
\text { Calculated from Sellmeier equation } \\
\text { Difference between observed and calculated values.- }\end{array}$ & $\begin{array}{c}1.515345 \\
37.4 \times 10^{-6} \\
1.515169 \\
+176 \times 10^{-6} \\
1.515281 \\
+64 \times 10^{-6}\end{array}$ & $\begin{array}{l}1.519093 \\
35.2 \times 10^{-6} \\
1.519138 \\
-45 \times 10^{-6} \\
1.519116 \\
-23 \times 10^{-6}\end{array}$ & $\begin{array}{l}1.522415 \\
63.6 \times 10^{-6} \\
1.522510 \\
-95 \times 10^{-6} \\
1.522420 \\
-5 \times 10^{-6}\end{array}$ & $\begin{array}{c}1.528536 \\
43.2 \times 10^{-6} \\
1.528757 \\
-221 \times 10^{-6} \\
1.528660 \\
-124 \times 10^{-6}\end{array}$ & $\begin{array}{l}1.536292 \\
49.3 \times 10^{-6} \\
1.536108 \\
+184 \times 10^{-6} \\
1.536202 \\
+90 \times 10^{-6}\end{array}$ & $\begin{array}{c}0.013193 \\
10.5 \times 10^{-6} \\
0.013588 \\
-395 \times 10^{-6} \\
0.013379 \\
-186 \times 10^{-6}\end{array}$ \\
\hline
\end{tabular}

It will be noted that the representation of the data is still not adequate, although the differences and standard deviations associated with the Sellmeier equation are appreciably less than the corresponding values associated with the Cauchy equation. The standard deviation associated with the variance shown in the table is $84 \times 10^{-6}$.

The corresponding Lorenz dispersion equation, which is quite similar to the Sellmeier form, is as follows:-

$$
\frac{n^{2}-1}{n^{2}+2}=\frac{C_{2}}{\nu_{0}^{2}-\nu^{2}}
$$

Preliminary calculations indicated that it did not represent the data as well as the Sellmeier equation. Consequently, it was not considered further.

The equations thus far presented have been two-constant equations. In no previous case with which we are familiar has a two-constant equation been adequate to express the dispersion shown by fifth-place refractive index measurements. A three-constant equation may be successful for some materials, but most of them require a fourconstant equation. However, it has not been found necessary to go beyond a four-constant equation. Such an equation, for example, has been shown to be quite adequate to represent the sixth-place index values obtained in the highest precision measurements on water [3, 14]. The Ketteler-Helmholtz equation has a theoretical justification more satisfactory than that behind the simpler equations, and probably should be used for the calculation of index at wavelengths other than those given here.

\section{Conclusions}

The refractive index of natural rubber and its variation with temperature and wavelength are those which would be predicted for a similar hydrocarbon of low molecular weight. A twoconstant equation of the Cauchy or Sellmeier type is inadequate to express the relation between refractive index and wavelength for rubber.

For convenient reference the best values resulting from the present investigation are recapitulated in table 6.

TABLE 6. "Best" values resulting from present invesitgation

\begin{tabular}{|c|c|c|c|c|c|c|}
\hline & $C$ & $D$ & $e$ & $F$ & $g$ & $F-C$ \\
\hline $\begin{array}{l}n^{25} \\
\text { Standard deviation of } n^{25} \\
d n / d T \\
\text { Standard deviation of } d n / d T\end{array}$ & $\begin{array}{r}1.515345 \\
37.4 \times 10^{-6} \\
-367.7 \times 10^{-6} \\
5.90 \times 10^{-6}\end{array}$ & $\begin{array}{r}1.519093 \\
35.2 \times 10^{-6} \\
-369.9 \times 10^{-6} \\
5.54 \times 10^{-6}\end{array}$ & $\begin{array}{r}1.522415 \\
63.6 \times 10^{-6} \\
-370.1 \times 10^{-6} \\
8.11 \times 10^{-6}\end{array}$ & $\begin{array}{r}1.528536 \\
43.2 \times 10^{-6} \\
-376.5 \times 10^{-6} \\
6.62 \times 10^{-6}\end{array}$ & $\begin{array}{r}1.536292 \\
49.3 \times 10^{-6} \\
-378.2 \times 10^{-6} \\
6.29 \times 10^{-6}\end{array}$ & $\begin{array}{r}0.013193 \\
10.5 \times 10^{-6} \\
-8.8 \times 10^{-6} \\
1.6 \times 10^{-6}\end{array}$ \\
\hline
\end{tabular}

\section{References}

[1] L. A. Wood, The optical properties of rubber, J. Applied Phys. 12, 119 (1941). Reprinted in Rubber Chem. Tech. 15, 23 (1942).

[2] L. W. Tilton, Permissible curvature of prism surfaces and inaccuracy of collimation in precise minimumdeviation refractometry, BS J. Research 11, 25 (1933).

Refractive Index of Rubber

$839743-49-5$
[3] L. W. Tilton and J. K. Taylor, Refractive index and dispersion of distilled water for visible radiation at temperatures $0^{\circ}$ to $60^{\circ} \mathrm{C}, \mathrm{J}$. Research NBS $\mathbf{2 0}$, 419 (1938) RP1085.

[4] G. W. Snedecor, Statistical methods (Iowa State College Press, Ames, Iowa, 1946).

[5] A. T. McPherson, A method for the purification of rubber and properties of the purified rubber, BS J. Research 8, 751 (1932) RP449. Reprinted in Rubber Chem. Tech. 5, 523 (1932). 
[6] A. T. MePherson and A. D. Cummings, Refractive index of rubber, J. Research NBS 14, 553 (1935) RP786. Reprinted in Rubber Chem. Tech. 8, 421 (1935).

[7] L. A. Wood, Values of the physical constants of rubber, Proc. Rubber Technology Conference, London, p. 933 (W. Heffer and Sons, Cambridge, Eng., 1938). Reprinted in Rubber Chem. Tech. 12, 130 (1939).

[8] F. Kirchhof, The refractometry of rubber and gutta percha hydrocarbons, Kautschuk 8, 137 (1932). Translated in Rubber Chem. Tech. 6, 92 (1933).

[9] N. Bekkedahl and L. A. Wood, Crystallization of vulcanized rubber, Ind. Eng. Chem. 33, 381 (1941). Reprinted in Rubber Chem. Tech. 14, 347 (1941).

[10] W. J. Taylor, J. M. Pignocco, and F. D. Rossini, Method for calculating the properties of hydrocarbons and its application to the refractive indices, densities, and boiling points of the paraffin and monoolefin hydrocarbons, J. Research NBS 34, 413 (1945) RP1651.

[11] S. S. Kurtz and A. G. Ward, The refractivity intercept and the specific refraction equation of Newton, J. Franklin Inst. 222, 563 (1936); 224, 583, 697 (1937)

[12] F. D. Rossini, K. S. Pitzer, W. J. Taylor, J. R. Ebert, J. E. Kilpatrick, C. W. Beckett, M. G. Williams, and H. G. Werner, Selected values of properties of hydrocarbons, NBS Circular C461 (1947).

[13] G. A. Jeffrey, Structure of polyisoprenes, I. The crystal structure of geranylamine hydrochloride. VI. An investigation of the molecular structure of dibenzyl by X-ray analysis, Proc. Roy. Soc. A183, 388 (1945); A188, 222 (1947).

[14] L. W. Tilton, Accurate representation of refractive index of distilled water as a function of wave length, J. Research NBS 17, 639 (1936) RP934.

Washington, March 11, 1949. 